\title{
Present Status of Neutron-, Photo-induced and Sponta- neous Fission Yields Experimental Data
}

\author{
B. Pritychenko ${ }^{1, *}, O$. Schwerer ${ }^{2}, J$. Totans ${ }^{1}$, and $O$. Gritzay $^{2}$ \\ ${ }^{1}$ National Nuclear Data Center, Brookhaven National Laboratory, Upton, NY 11973-5000, USA \\ ${ }^{2}$ Under contract with National Nuclear Data Center, Brookhaven National Laboratory, Upton, NY \\ 11973-5000, USA
}

\begin{abstract}
Nuclear reaction data collection, evaluation and dissemination have been pioneered at the Brookhaven National Laboratory since the early 50s. These activities gained popularity worldwide, and around 1970 the experimental nuclear reaction data interchange or exchange format (EXFOR) was established. The original EXFOR compilation scope consisted only of neutron reactions and spontaneous fission data, while many other nuclear data sets were ignored. Due to the high cost of new experiments, it is very important to find and recover the previously disregarded data using scientific publications, data evaluations and nuclear databases comparisons.

Fission yields play a very important role in applied and fundamental physics, and such data are essential in many applications. The comparative analysis of Nuclear Science References (NSR) and Experimental Nuclear Reaction (EXFOR) databases shows a large number of unaccounted experiments and provides a guide for the recovery of fission cross sections, yields and covariance data sets. The dedicated fission yields data compilation effort is currently underway in the Nuclear Reaction Data Centers (NRDC) network, and includes identification, compilation, storage and Web dissemination of the recovered data sets.
\end{abstract}

\section{Introduction}

The early success of Brookhaven National Laboratory (BNL) reactor program which generated large volumes of new results showed a strong need for data preservation and dissemination. Compilation of neutron cross sections have been initiated at BNL in the early 1950s by Donald Hughes (1915-1960). The Brookhaven group produced the famous BNL-170 report [1]. The Brookhaven report was a successor of the Metallurgical Laboratory, University of Chicago neutron cross section compilation [2,3], and it is currently on display at the Donald Hughes book library as shown in Fig. 1. The National Nuclear Data Center (NNDC) book library is the major resource behind the NSR and EXFOR database contents development. Several nuclear reaction data projects such as the Atlas of Neutron Resonances reference book [4], Evaluated Nuclear Data File (ENDF) [5] and the Experimental Nuclear Reaction Data (EXFOR) $([6,7]$ and references therein) predecessor and the SCISRS (Sigma Center Information Storage and Retrieval System) system [8] take their roots from the Brookhaven cross section compilations.

\footnotetext{
*e-mail: pritychenko@bnl.gov
} 


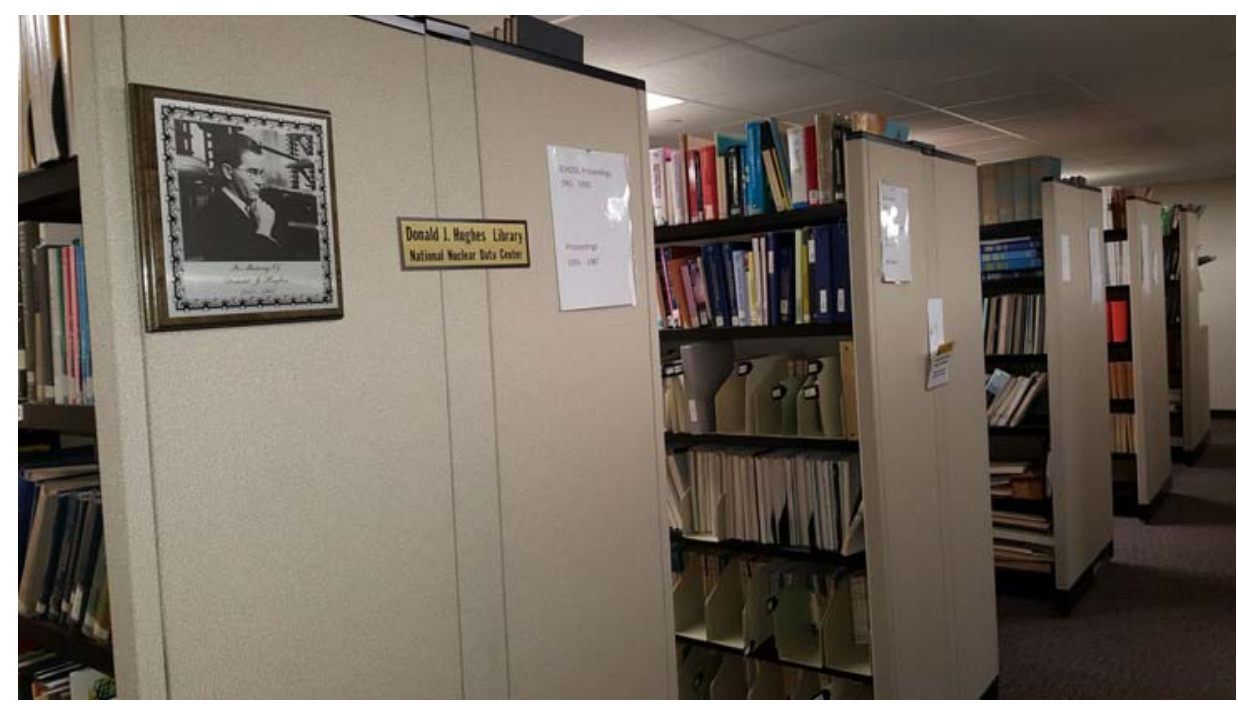

Figure 1. Donald Hughes book library at the National Nuclear Data Center, Brookhaven National Laboratory.

Over the years the data compilation efforts have evolved and spread worldwide which was facilitated by the nuclear data developments in Vienna, Austria (Nuclear Data Section or NDS-IAEA), Paris, France (Nuclear Energy Agency or NEA-Databank) and Obninsk, USSR (Center Jadernykh Dannykh or CJD) in 1963-1964. In 1970 the four original data centers agreed on the data interchange or exchange format (EXFOR). This agreement led to the formation of the Nuclear Reaction Data Centres (NRDC) network under the auspices of the International Atomic Energy Agency (IAEA) to oversee the neutron data compilations, and the NRDC will celebrate its golden jubilee in 2020. Authority for reaction data compilations were assigned on a geographical basis: Area \#1: U.S. and Canada (NNDC), Area \#2 Western Europe + Japan (NEA-Databank), Area \#3 Eastern Europe, Africa, Asia, Australia, Latin America (NDS-IAEA) and Area \#4: Former USSR (CJD).

The geographical separation of compilation work has simplified interactions between researchers and data compilers. This joint effort resulted in a successful international collaboration and produced the only continuously-updated low- and intermediate-energy nuclear reaction EXFOR database, and these data are publicly available from the NDSIAEA (https://www-nds.iaea.org/exfor/) or NNDC (https://www.nndc.bnl.gov/exfor/) web sites. Fig. 2 highlights the distribution of $\sim 18$ million data points supplied to EXFOR by 12 active and 3 discontinued centers. The database major contributors include NNDC (Brookhaven, USA), NEA-DB (OECD, Paris, France), NDS (IAEA, Vienna, Austria), CNPD (Sarov, Russian Federation) and JCPRG (Sapporo, Japan). The graphic data show that the majority of complex measurements were performed in the USA and Europe, and NNDC (Area \#1) and NEA-Databank (Area \#2), NDS-IAEA (Area \#3) provide the largest individual contribution to the library. These centers are closely followed by the data efforts in the Russian Federation and Japan. 


\section{EXFOR Compilation Statistics: Data Points}

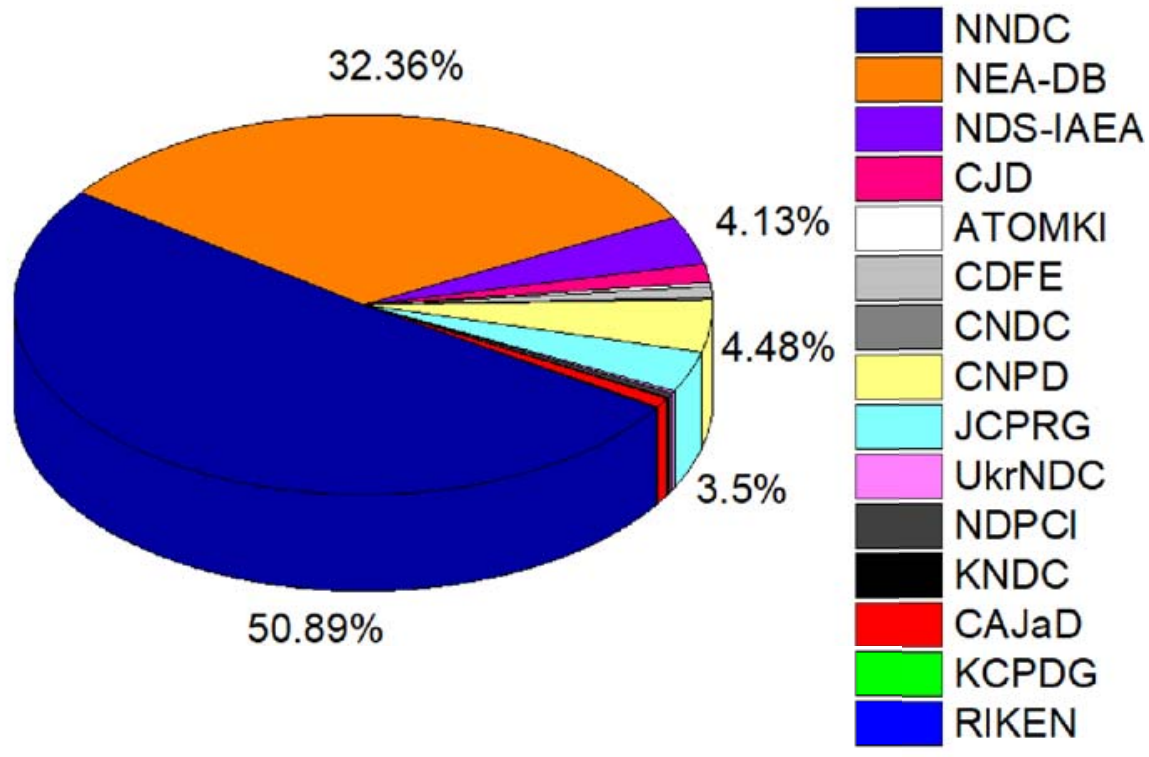

Figure 2. EXFOR compilation statistics include data points from the currently operating NNDC Brookhaven, NEA-DB Paris, NDS IAEA Vienna, CJD Obninsk, ATOMKI Debrecen, CDFE Moscow, CNDC Beijing, CNPD Sarov, JCPRG Sapporo, UkrNDC Kyiv, NDPCI BARC/India, KNDC Taejon and discontinued CAJaD, KCPDG and RIKEN data centers. Complete centers descriptions and complementary details are available from the EXFOR compilation control system: https://wwwnds.iaea.org/public/exfor/x4compil/. As of November 11, 2019 the total number of data points was $17,467,585$.

\section{Missing Data}

Today, EXFOR library and its computer database are primary repository of experimental neutron, charged-particle, and photonuclear reaction data compilations. However, the broad scope of compilations was not a case at the beginning, and the library has evolved due to historical and technological reasons. Fig. 3 shows that the initial compilation area was focused only on neutron cross section and spontaneous fission data while many important nuclear physics quantities such as neutron fission yields, charged-particle and photonuclear reaction parameters were not compiled until the compilation scope was changed in 1976. In those days, computer technologies were very immature and required large number of people to produce the relatively small compilations based on tabulated data. The lack of Internet access and general unavailability of laboratory reports [9] produced a situation wherein many important results were unnoticed by the EXFOR compilers. These missing EXFOR references were analyzed in our previous publication [10], and the results are shown graphically in Fig. 4.

To further understand the situation we would consider the case of ${ }^{254} \mathrm{Cf}$ spontaneous fission. Tentative observations of the heavy element production in neutron stars merger (GW010817) [11-13] created plenty of enthusiasm in the nuclear astrophysics community, and several complementary scenarios were developed. One of the most interesting possibilities includes analysis of Kilonova light curves for the possible impact of ${ }^{254} \mathrm{Cf}$ spontaneous 


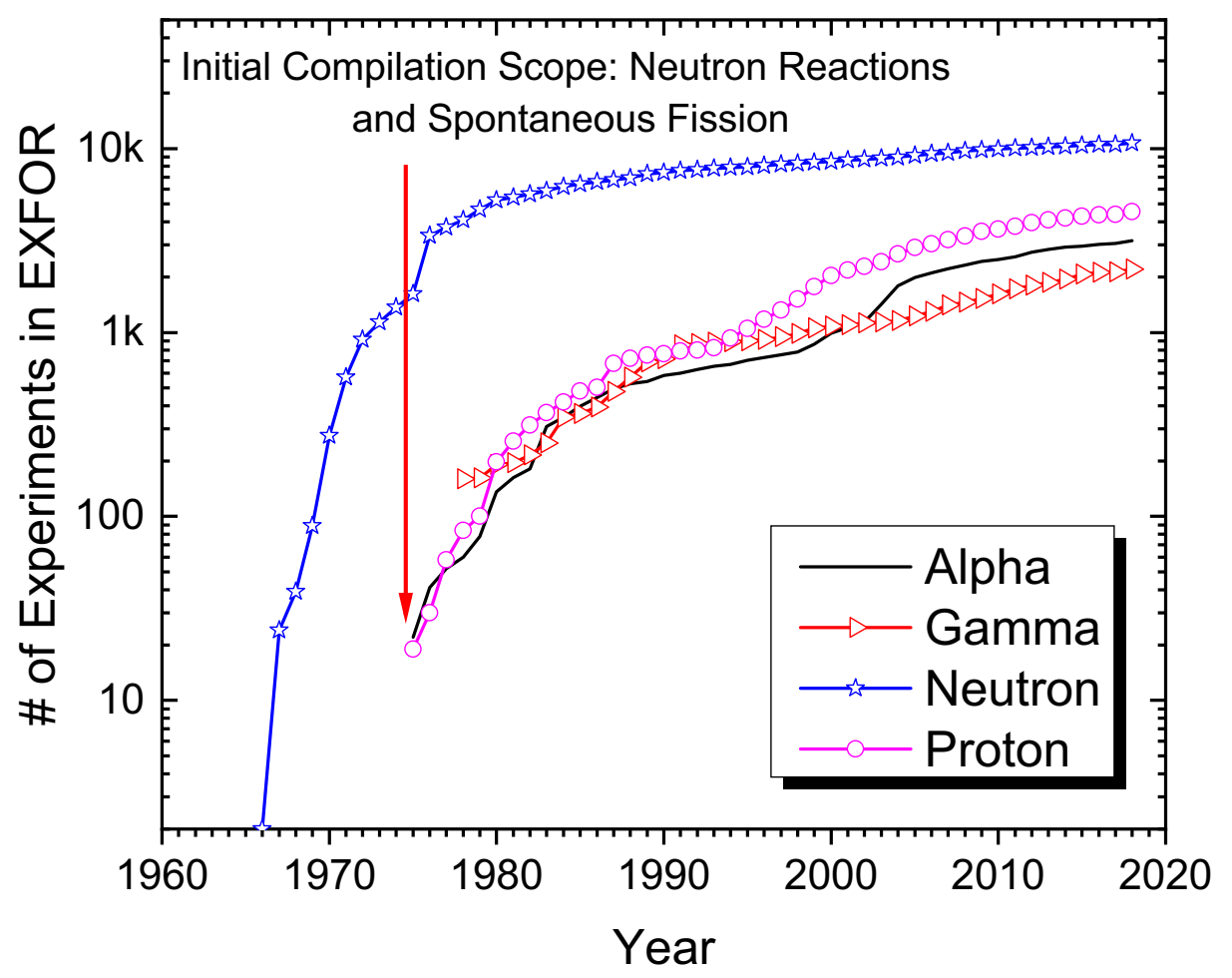

Figure 3. EXFOR compilation timeline shows evolution of the library compilation scope and data contents over the years.

fission [14]. The authors of this paper could not find relevant ${ }^{254} \mathrm{Cf}$ spontaneous fission data using the EXFOR database [6] and resorted to theoretical calculations. However, the decays of the californium isotopes have been extensively studied by J. Wilhelmy's group at Los Alamos National Laboratory. In fact, the substantial amount of ${ }^{254} \mathrm{Cf}\left(\sim 10^{12}\right.$ nuclei) has been produced at the TRU (trans-uranium) facility at Oak Ridge National Laboratory as part of the trans-uranium production programme of Energy Research and Development Administration (ERDA) for a USA /Israel group. The results were produced at the Weizmann Institute, Rehovot, Israel and published as the Grenoble conference proceedings and a Jülich, Germany laboratory report [15-17]. It was very common in the 70s when scientists published their findings in secondary publications [9] that were not accessible to EXFOR compilers. The recent publication of Kilonova light curves analysis [14] has motivated the nuclear data community to re-examine this situation using complementary resources. The analysis of the Nuclear Science References database [18] by searching for ${ }^{254} \mathrm{Cf}$ experimental data reveals several experiments $[16,17]$ in which spontaneous fission of ${ }^{254} \mathrm{Cf}$ was extensively studied. These previously missed fission yields were presented to the astrophysics community and experimental data compilations are in progress.

\section{Analysis of Fission Yields}

It is common knowledge that any new measurements are very expensive. For instance, the cost of irradiation of electronic components for space flights at the 88" Lawrence Berkeley 


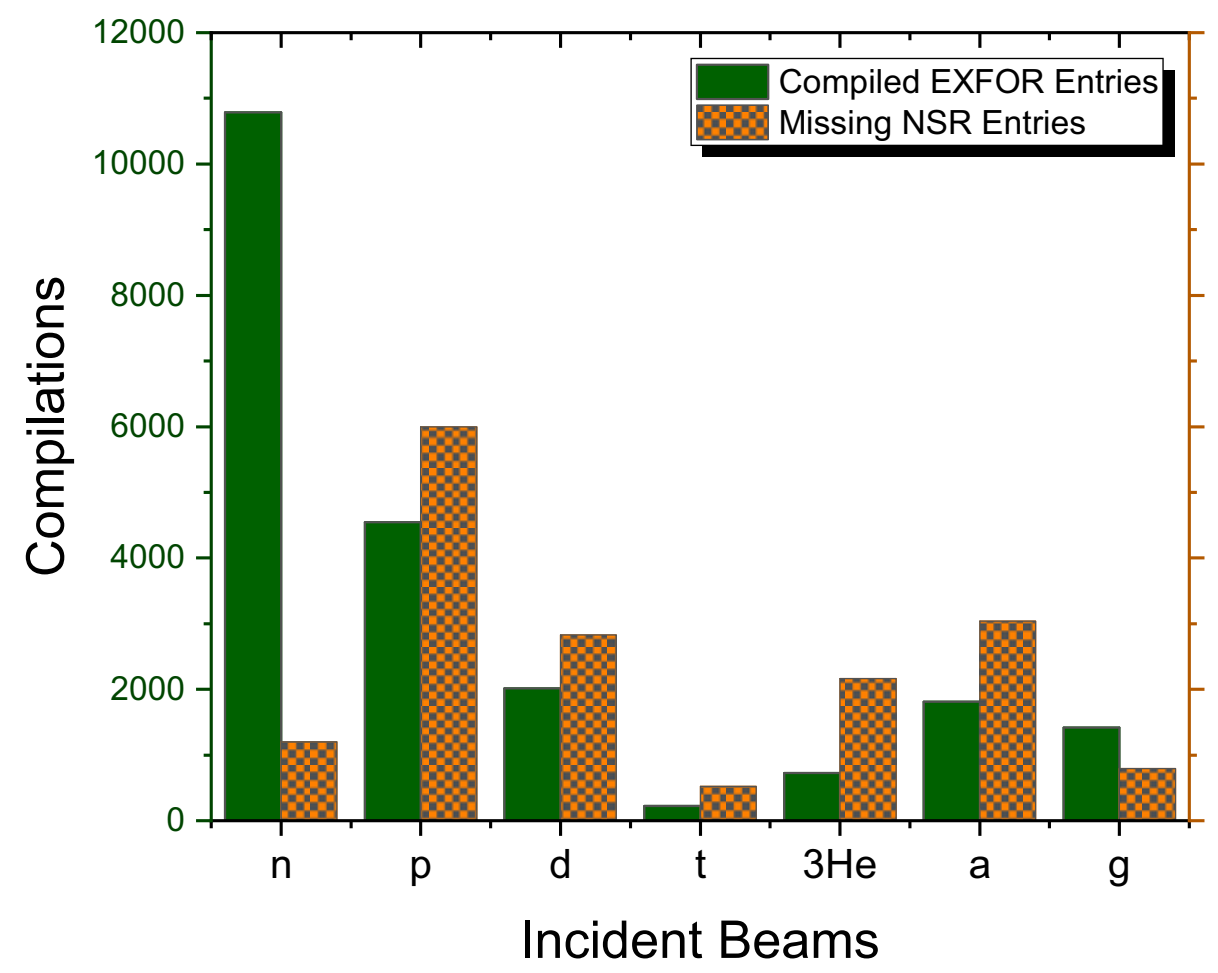

Figure 4. EXFOR completeness for neutron-, charged- and photonuclear-induced reactions.

National Laboratory is $\$ 2,500$ per hour [19]. This results in the $\$ 420 \mathrm{~K}$ beam time price tag for a typical one-week experiment. Addition of the target, electronics and staff expenses brings the overall cost to $\$ 1 \mathrm{M}$. Therefore, it is very cost effective to recover the previouslypublished results before conducting a new measurement. Further analysis allows us to conservatively estimate the monetary value of $\sim 23,000$ EXFOR experiments is approximately $\$$ $23 \mathrm{~B}$. This finding clearly demonstrates the importance of the present work and the strong needs for nuclear reaction data preservation, storage and dissemination.

Recently, the overall situation with missing in EXFOR fission yields compilations has been analyzed at the NNDC using the NSR database that is based on the Donald Hughes book library [10]. These results were summarized in three NRDC memos [20-23] that provided a guide for the network to fix the EXFOR database deficiencies. A similar effort is currently underway at the Nuclear Data Section, IAEA, Vienna [24]. The IAEA group has analyzed references from the U.S. and U.K. fission yields evaluations [25, 26]. This complementary effort would ensure the completeness of the EXFOR database for fission data. In addition, the fission yield evaluation references $[25,26]$ were explored at NNDC for missing in NSR articles, PDF files were collected, and publications were entered into the database. These developments guarantee the mutual completeness of the NSR and EXFOR databases that is needed for nuclear reaction and structure evaluations.

The missing data analysis has been used to estimate the workload for the Area \#1 fission yields compilations that are now in progress. By the end of Fiscal Year 2019, 18 new neutron, 6 new photo-induced fission and 41 revised neutron-induced fission compilations have been submitted to the NRDC network. The compilation of previously missed photofission work 
of Smith and Richardson [27] is shown in Fig. 5. The new reaction entry consists of one bibliographical and two data subentries. The first data subentry contains independent fission yields for isotopes of $\mathrm{Br}, \mathrm{Rb}, \mathrm{Nb}, \mathrm{Sb}$ and $\mathrm{Cs}$. The second data subentry contains chain fission yields for $A=125,127$. These data have been processed in EXFOR format using the Sarov EXFOR editor [28]. The NNDC compilation project is currently going through the Brookhaven group memos [20-23]. Work on the IAEA group findings [24] should start in 2020, and the tentative target completion is Fall 2020.

\section{Conclusion}

The current work reviews the present status of fission yields experimental data and describes compilation work in Area \#1. The missing database references have been identified and corresponding PDF files for the articles were recovered. These materials were shared with the NRDC network, and the compilation activities are currently underway at the NNDC. The examples of the NSR database queries for missing references, data analyses, and compilations are given. NSR and EXFOR databases comparison demonstrated the large potential of both databases and produced many complementary findings. The results of this work are an integral part of the BNL-Los Alamos reevaluation of neutron fission yields [29] that would make a broad impact in multiple areas of applied and fundamental science.

The authors are indebted to A. Sonzogni (BNL) for encouragement of this project, T. Kawano (LANL) for useful comments and R. Arcilla (BNL) for a careful reading of the manuscript and valuable suggestions. We are grateful to the NRDC network members for the continuous work on EXFOR data compilations and overall database improvements. We acknowledge partial support from the NA-22 grant number 000024158. Work at Brookhaven was funded by the Office of Nuclear Physics, Office of Science of the U.S. Department of Energy, under Contract No. DE-AC02-98CH10886 with Brookhaven Science Associates, LLC.

\section{References}

[1] D.J. Hughes, T.W. Bonner, H. Goldstein, W.W. Havens, Jr., I. Kaplan, C.O. Muehlhause, A.H.Snell, J.R. Stehn, T.M. Shyder, R.F. Taschek, A. Wattenberg, "A Compilation of the AEC Neutron Cross Section Advisory Group," Brookhaven National Laboratory Report 170, May 15 (1952).

[2] H.H. Goldsmith, W.W. Ibser, "Neutron Cross Sections of the Elements," United States Atomic Energy Commission Report, MMDC 59 (1946).

[3] H.H. Goldsmith, W.W. Ibser, B.T. Feld, "Neutron Cross Sections of the Elements A Compilation," Review of Modern Physics 19, 259 (1947).

[4] S.F. Mughabghab, Atlas of Neutron Resonances, Resonance Properties and Thermal Cross Sections Z=1-60 1, Elsevier Publisher, Amsterdam (2018).

[5] D.A. Brown, M.B. Chadwick, R. Capote, A.C. Kahler, A.Trkov, M.W. Herman, A.A. Sonzogni, Y. Danon, A.D. Carlson, M. Dunn, D.L. Smith, G.M. Hale, G. Arbanas, R. Arcilla, C.R. Bates, B. Beck, B. Becker, F. Brown, R.J. Casperson, J. Conlin, D.E. Cullen, M.-A. Descalle, R. Firestone, T. Gaines, K.H. Guber, A.I. Hawari, J. Holmes, T.D. Johnson, T. Kawano, B.C. Kiedrowski, A.J. Koning, S. Kopecky, L. Leal, J.P. Lestone, C. Lubitz, J. I.Marquez Damian, C.M. Mattoon, E.A. McCutchan, S. Mughabghab, P. Navratil, D. Neudecker, G.P.A. Nobre, G. Noguere, M.Paris, M.T. Pigni, A.J. Plompen, B. Pritychenko, V.G. Pronyaev, D. Roubtsov, D. Rochman, P.Romano, P. Schillebeeckx, S. Simakov, M. Sin, I. Sirakov, B. Sleaford, V. Sobes, E.S. Soukhovitskii, I. Stetcu, P. Talou, I. Thompson, 
S. van der Marck, L. Welser-Sherrill, D. Wiarda, M. White, J. L.Wormald, R. Q.Wright, M. Zerkle, G. Zerovnik, Y. Zhu, Nucl. Data SheEts 148, 1 (2018).

[6] V.V. Zerkin, B. Pritychenko, "The experimental nuclear reaction data (EXFOR): Extended computer database and Web retrieval system," Nucl. Instrum. Meth. Phys. Res. A 888, 31 (2018).

[7] N. Otuka, E. Dupont, V. Semkova, B. Pritychenko, A.I. Blokhin, M. Aikawa, S. Babykina, M. Bossant, G. Chen, S. Dunaeva, R.A. Forrest, T. Fukahori, N. Furutachi, S. Ganesan, Z. Ge, O.O. Gritzay, M. Herman, S. Hlavac, K. Kato, B. Lalremruata, Y.O. Lee, A. Makinaga, K. Matsumoto, M. Mikhaylyukova, G. Pikulina, V.G. Pronyaev, A. Saxena, O. Schwerer, S.P. Simakov, N. Soppera, R. Suzuki, S. Takacs, X. Tao, S. Taova, F. Tarkanyi, V.V. Varlamov, J. Wang, S.C. Yang, V. Zerkin, Y. Zhuang, “Towards a More Complete and Accurate Experimental Nuclear Reaction Data Library (EXFOR): International Collaboration between Nuclear Reaction Data Centres (NRDC)," Nucl. Data ShEETs 120, 272 (2014).

[8] N. Holden, "A Short History of CSISRS: At the Cutting Edge of Nuclear Data Information Storage and Retrieval Systems and its Relationship to CINDA, EXFOR and ENDF," December 2005. Downloaded from 〈https://www.nndc.bnl.gov/exfor/compilations/ CSISRSHistory.pdf $\rangle$ on June 26, 2019.

[9] B. Pritychenko, "Evolving landscape of low-energy nuclear physics publications," ScIENTOMETRICs 109, 2067 (2016).

[10] B. Pritychenko, O.Schwerer, J. Totans, V. Zerkin, O. Gritzay, "Completeness of Neutron-, Photo-induced and Spontaneous Fission Yields Data," Eur. Phys. J. Cof. (2020).

[11] E. Pian, P. D’Avanzo, S. Benetti, et al., Nature(London) 551, 67 (2017).

[12] S.J. Smartt, T.-W. Chen, A. Jerkstrand, et al., Nature(London) 551, 75 (2017).

[13] N.R. Tanvir, A.J. Levan, C. Gonzalez-Fernandez, et al., Astrophys. J. 848, L27 (2017).

[14] Y. Zhu, R.T. Wollaeger, N. Vassh, R. Surman, T.M. Sprouse, M.R. Mumpower, P. Möller, G.C. McLaughlin, O. Korobkin, T. Kawano, P.J. Jaffke, E.M. Holmbeck, C.L. Fryer, W.P. Even, A.J. Couture, J. Barnes, "Californium-254 and Kilonova Light Curves," Astrophys.J. 863, L23 (2018).

[15] E. Cheifetz, H.A. Selic, A. Wolf, J.B. Wilhelmy, "Even-Even Neutron-Rich Isotopes," Contrib. Conf. Nucl. Spectrosc. Fission Products, Grenoble, 193 (1979).

[16] H.A. Selic, E. Cheifetz, A. Wolf, J.B. Wilhelmy, "Neutron-Rich Fragments from Spontaneous Fission of ${ }^{254}$ Cf," Contrib. Conf. Nucl. Spectrosc. Fission Products, Grenoble, 316 (1979).

[17] H.A. Selic, E. Cheifetz, J.B. Wilhelmy, "Studies of Primary Fission Products from ${ }^{254}$ Cf," JUL-Spez-99, 69 (1981).

[18] B. Pritychenko, E. Betak, M.A. Kellett, B. Singh, J. Totans, "The Nuclear Science References (NSR) database and Web Retrieval System," Nucl. Instr. Meth. Phys. Res. A 640, 213 (2011).

[19] L. Bernstein, private communication to B. Pritychenko, March (2019).

[20] O. Schwerer, B. Pritychenko, "Completeness check EXFOR vs. NSR: Spontaneous Fission Yields," NRDC Memo CP-C/464, NDS-IAEA, 29 November 2018.

[21] O. Schwerer, B. Pritychenko, "Completeness check EXFOR vs. NSR: Photofission Yields," NRDC Memo CP-C/465, NDS-IAEA, 30 January 2019.

[22] O. Schwerer, B. Pritychenko, "Completeness check EXFOR vs. NSR: Neutron-induced fission yields," NRDC Memo CP-C/466, NDS-IAEA, 19 February 2019.

[23] O. Schwerer, "Procedure used for the completeness check EXFOR vs. NSR on fission yields," NRDC Memo CP-C/469, NDS-IAEA, 14 April 2019. 
[24] S. Okumura, N. Otuka, et al., Eur. Phys. J. Cof. (2020).

[25] T.R. England, B.F. Rider, "Evaluation and Compilation of Fission Product Yields 1993," Los Alamos National Laboratory Report LA-UR-94-3106 (1994).

[26] R.W. Mills, "Fission product yield evaluation," Thesis, Univ. of Birmingham (1995).

[27] J.R. Smith, A.E. Richardson, "Independent yields from the photofission of 232Th and the Z(p) and statistical-dynamic models," Phys.Rev. C 44, 1118 (1991).

[28] G.N. Pikulina, S.M. Taova, "Activities of the RFNC-VNIIEF Center of Nuclear Physics Data on the Compilation of Experimental Data for the EXFOR International Library: EXFOR-EDITOR Software Package," Phys.Atomic Nuclei 81, 1450 (2018).

[29] "Nuclear Data Interagency Working Group / Research Program," DOE National Laboratory Announcement Number: LAB 18-1903. Downloaded from 〈https://www.energy.gov/science/np/nuclear-physics〉 on June 26, 2019. 


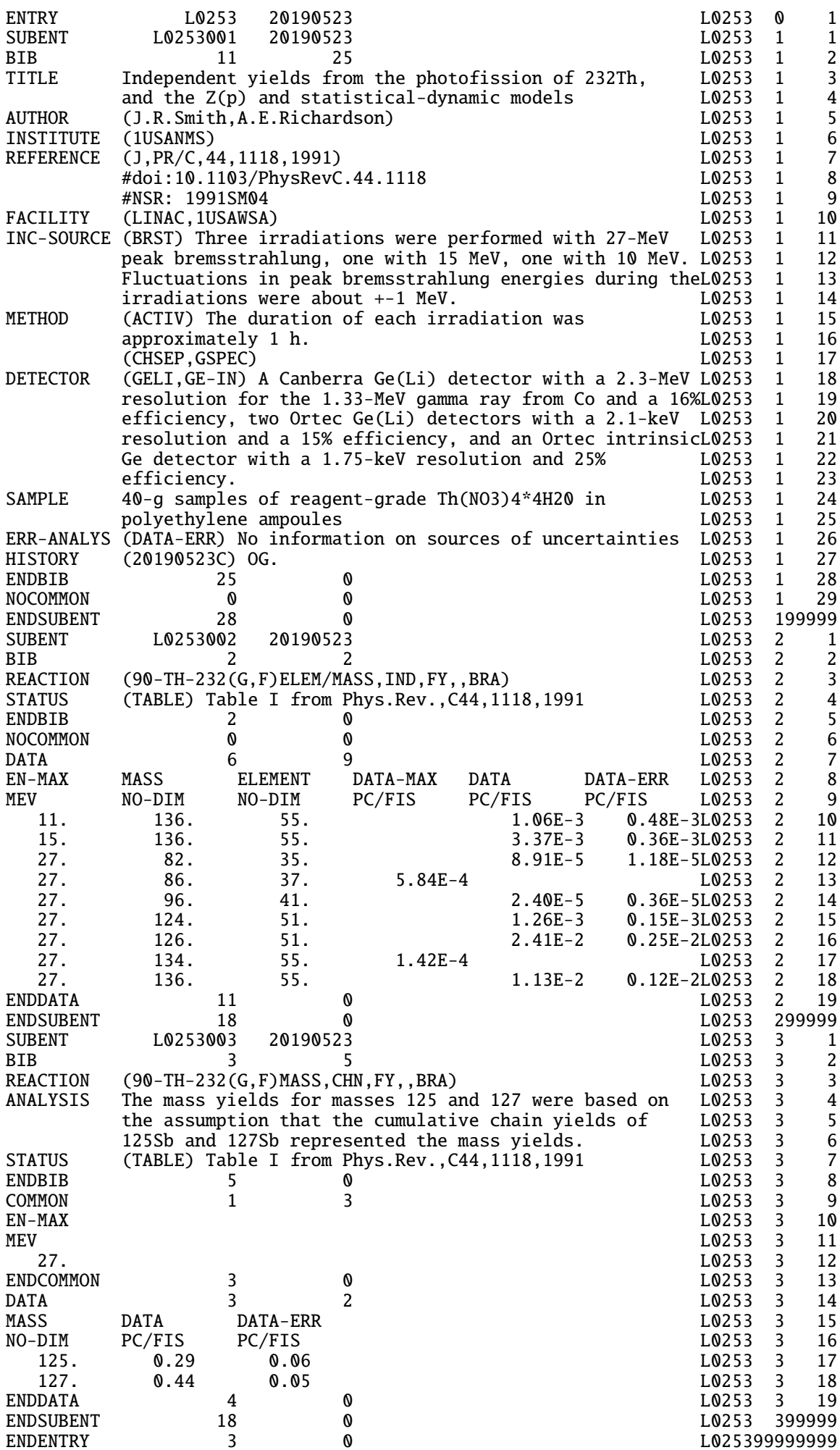

Figure 5. EXFOR compilation of ${ }^{232} \mathrm{Th}(\gamma, \mathrm{F})$ work of J.R. Smith and A.E. Richardson [27]. 